\title{
Ricci surfaces
}

\author{
Andrei Moroianu ANd SERgIU Moroianu
}

\begin{abstract}
A Ricci surface is a Riemannian 2-manifold $(M, g)$ whose Gaussian curvature $K$ satisfies $K \Delta K+g(d K, d K)+4 K^{3}=0$. Every minimal surface isometrically embedded in $\mathbb{R}^{3}$ is a Ricci surface of non-positive curvature. At the end of the $19^{\text {th }}$ century Ricci-Curbastro has proved that, conversely, every point $x$ of a Ricci surface has a neighborhood which embeds isometrically in $\mathbb{R}^{3}$ as a minimal surface, provided $K(x)<0$. We prove this result in full generality by showing that Ricci surfaces can be locally isometrically embedded either minimally in $\mathbb{R}^{3}$ or maximally in $\mathbb{R}^{2,1}$, including near points of vanishing curvature. We then develop the theory of closed Ricci surfaces, possibly with conical singularities, and construct classes of examples in all genera $g \geq 2$.
\end{abstract}

Mathematics Subject Classification (2010): 49Q05 (primary); 53C27, 53C42 (secondary).

\section{Introduction}

In 1873 Ludwig Schläfli asked the following question, still unanswered today (cf. [28,29]):

Can every Riemannian surface $\left(M^{2}, g\right)$ be locally isometrically embedded in the flat space $\mathbb{R}^{3}$ ?

The problem reduces to a non-linear equation of Monge-Ampère type. This equation can be easily solved near points $x$ where the Gaussian curvature $K(x)$ is nonvanishing, but it is degenerate at points where $K(x)=0$. A partial positive answer was recently obtained provided that the gradient of the Gaussian curvature has a special behavior in the neighborhood of the zero set of $K$ (see [10] and references therein).

Partially supported by the ANR-10-BLAN 0105 grant of the Agence Nationale de la Recherche. Partially supported by the LEA "MathMode" and the CNCS grant PN-II-RU-TE-2012-3-0492.

Received June 18, 2012; accepted in revised form November 25, 2013. 
A related question was asked in 1895 by Gregorio Ricci-Curbastro [18] about minimal embeddings in $\mathbb{R}^{3}$ :

When does a Riemannian surface $\left(M^{2}, g\right)$ carry minimal local isometric embeddings in the flat space $\mathbb{R}^{3}$ ?

The answer is known near points of non-zero Gaussian curvature:

Theorem 1.1 ([18], [4, page 124]). A Riemannian surface $\left(M^{2}, g\right)$ with negative Gaussian curvature $K<0$ has local isometric embeddings as minimal surface in the flat space $\mathbb{R}^{3}$ if and only if one of the two equivalent conditions below holds:

(i) The metric $\sqrt{-K} g$ is flat;

(ii) The Gaussian curvature satisfies

$$
K \Delta K+g(d K, d K)+4 K^{3}=0
$$

where $\Delta=\delta^{g} d$ denotes the scalar Laplace operator of the metric $g$.

Condition (i) is usually referred to as the Ricci condition. This condition does not hold in general for minimal surfaces in $\mathbb{R}^{n}$ for $n \geq 4$, see $[13,17]$. In [26] Vlachos obtains some necessary conditions for the existence of local minimal immersions of $\left(M^{n}, g\right)$ in $\mathbb{R}^{n+p}$ for all $n \geq 2$ and $p \geq 1$. A generalization of Theorem 1.1 to pluriharmonic submersions of Kähler manifolds $\left(M^{2 n}, g, J\right)$ in $\mathbb{R}^{2 n+1}$ was obtained by Furuhata [9].

Our main result is the extension of Theorem 1.1 to the general case, with no assumption on the Gaussian curvature. Of course, the Ricci condition (i) no longer makes sense at points where $K$ vanishes, but we simply use the Ricci condition (ii) instead. It turns out that if $K$ satisfies (1.1), then it either vanishes identically, or does not change sign on $M$. Both signs might appear, and they correspond to minimal immersions in the Euclidean space and, respectively, to maximal immersions in the Lorentz space:

Theorem 1.2. Let $\left(M^{2}, g\right)$ be a connected Riemannian surface whose Gaussian curvature $K$ satisfies (1.1). Then $K$ does not change its sign on $M$. If $K \leq 0$, then $M$ can be locally isometrically immersed in $\mathbb{R}^{3}$ as a minimal surface. If $K \geq 0$, then $M$ can be locally isometrically immersed in the Lorentz space $\mathbb{R}^{2,1}$ as a maximal surface.

Using the spinorial characterization of isometric embeddings of surfaces in $\mathbb{R}^{3}$ or $\mathbb{R}^{2,1}$, a significant step in the proof of this theorem reduces to a statement formulated only in terms of holomorphic and harmonic functions on $\mathbb{C}$.

Theorem 1.3. Let $\Omega \subset \mathbb{C}$ be a simply connected domain and $F \in \mathcal{C}^{\infty}(\Omega, \mathbb{R})$. Assume that $\log |F|$ is harmonic at every point where $F \neq 0$. Then $F$ does not change sign, and there exists a holomorphic function $h$ with $|F|=|h|^{2}$. 
The main difficulty in Theorem 1.3 is to show that the zeros of $F$ are isolated. This is accomplished in Theorem 4.6 below, using ideas stemming from potential theory. We assume that the function $F$ has some non-isolated zeros. We prove in Lemma 4.7 that at its non-isolated zeros $F$ vanishes to infinite order. Then we show that the connected components of the complement of the set of non-isolated zeros cannot be simply connected, so there exist simple closed curves avoiding the zero set of $F$ and confining some non-isolated zeros of $F$. For every simple closed curve $\gamma$ on which $F$ does not vanish, we define a "virtual measure" of the zero set of $F$ lying in the region $\Omega$ bounded by $\gamma$. For example, in the case where $\log (F)$ is defined by convolution of the Green kernel of the Laplacian with a measure $\mu$ supported on some compact set $C$, the zero set of $F$ is $C$ and the virtual measure of $\Omega$ is just $\mu(C)$. The main properties of the virtual measure are positivity (Lemma 4.9) and additivity. To obtain a contradiction we divide $F$ by a sufficiently large power $n$ of the distance function to a non-isolated zero. We obtain again a smooth non-negative function whose logarithm is harmonic outside the zero-set and whose virtual measure decreases by $2 \pi n$ compared to that of $F$, thus contradicting the positivity of the virtual measure. This proof is carried out in detail in Section 4.

In a second part of the paper we study the existence and uniqueness question, in a given conformal class, of metrics satisfying the Ricci condition, also called Ricci metrics. We construct Ricci metrics of non-positive (respectively non-negative) curvature from spherical (respectively hyperbolic) metrics with conical points of angles integer multiples of $2 \pi$. For non-positive curvature we get for instance that every hyperelliptic surface of odd genus admits a Ricci metric. In the non-negative case, on a closed surface there exist conical Ricci metrics of positive curvature with prescribed conical singularities. These results are grouped in Section 6.

For the convenience of those readers more familiar with the classical viewpoint of minimal surfaces, we describe in the Appendix the link between our approach and the standard Weierstrass-Enneper representation.

The theory of minimal surfaces, although more than two centuries old, is still a very active field of research, and it is somehow surprising that the intrinsic characterization of minimal surfaces in $\mathbb{R}^{3}$ obtained here was only available so far in the case of non-vanishing Gaussian curvature. For the analytical aspects of minimal surfaces we refer to the recent monograph by Colding and Minicozzi [5]. From the huge literature in the subject, we would like to single out Taubes' recent study [21] of the moduli space of minimal surfaces embedded in $\mathbb{H}^{3}$, and Weber and Wolf's construction [27] of embedded minimal surfaces in $\mathbb{R}^{3}$ using the notion of orthodisks, which seems to be somewhat related to our method of constructing compact Ricci surfaces in Section 6 below.

ACKNOWLEDGEMENTS. We have benefited from many enlightening discussions with Christophe Margerin. His suggestions coming from potential theory inspired us the key ideas used in the proof of Theorem 4.6. 


\section{Preliminaries}

\subsection{Conformal metric changes on surfaces}

We start by recalling some well-known facts in conformal geometry. Assume that $g_{0}$ and $g:=e^{-2 f} g_{0}$ are Riemannian metrics on a surface $M$. Let $\Delta=\delta^{g} d$ and $K$, respectively $\Delta_{0}=\delta^{g_{0}} d$ and $K_{0}$, denote the Laplacian and the Gaussian curvature of $g$ and $g_{0}$. Then the following formulas hold ( $c f$. [3, page 59]):

$$
\begin{aligned}
& \Delta=e^{2 f} \Delta_{0}, \\
& K=e^{2 f}\left(K_{0}-\Delta_{0} f\right) .
\end{aligned}
$$

If we fix a spin structure and denote by $D$ and $D_{0}$ the Dirac operators corresponding to $g$ and $g_{0}$ respectively, then Hitchin's classical conformal covariance relation reads

$$
D \psi=e^{\frac{3 f}{2}} D_{0}\left(e^{-\frac{f}{2}} \psi\right)
$$

\subsection{Ricci surfaces}

Motivated by Ricci-Curbastro's local characterization of minimal surfaces in $\mathbb{R}^{3}$ (Theorem 1.1), we make the following:

Definition 2.1. A Riemannian surface $(M, g)$ whose Gaussian curvature $K$ satisfies the identity (1.1)

$$
K \Delta K+g(d K, d K)+4 K^{3}=0
$$

is called a Ricci surface, and $g$ is called a Ricci metric.

As mentioned in the introduction, Ricci metrics have several nice characterizations near points where the Gaussian curvature is negative:

Lemma 2.2. Let $(M, g)$ be a Riemannian surface with negative curvature $K<0$. The following four conditions are equivalent:

- $g$ is a Ricci metric;

- $\Delta \log (-K)+4 K=0$;

- the metric $(-K)^{1 / 2} \mathrm{~g}$ is flat;

- the metric $(-K) g$ is spherical, i.e., of constant Gaussian curvature 1.

Proof. We compute directly $\Delta \log (-K)+4 K=K^{-2}\left(K \Delta K+|d K|^{2}+4 K^{3}\right)$, hence the first two conditions are equivalent. For $r \in \mathbb{R}$ set $g_{r}:=(-K)^{r} g$. By Eq. (2.2), the Gaussian curvature $K_{r}$ of the metric $g_{r}$ equals

$$
K_{r}=(-K)^{-r}\left(K+\frac{1}{2} \Delta\left(\log (-K)^{r}\right)\right) .
$$


Assuming $\Delta \log (-K)+4 K=0$ we get $K_{r}=(1-2 r)(-K)^{-r} K$, hence $g_{1 / 2}$ is flat and $g_{1}$ has constant Gaussian curvature equal to 1 . Conversely, if $K_{1 / 2}=0$ then (2.4) for $r=\frac{1}{2}$ implies $\Delta \log (-K)+4 K=0$, and the same conclusion holds if $K_{1}=1$.

We thus see that the conformal class of a negatively curved Ricci metric contains both a flat and a round metric. Conversely, we can construct Ricci metrics in any conformal class known a priori to contain both a spherical and a flat metric:

Lemma 2.3. Let $g_{1 / 2}$ be a flat metric on a surface $M$ and $V \in \mathcal{C}^{\infty}(M), V>0$ such that $g_{1}:=V g_{1 / 2}$ is spherical. Then $g:=V^{-1} g_{1 / 2}$ is a Ricci metric of curvature $-V^{2}$.

Proof. Denote by $K, K_{1 / 2}, K_{1}$ and $\Delta, \Delta_{1 / 2}, \Delta_{1}$ the Gaussian curvatures and the Laplacians of $g, g_{1 / 2}$, respectively $g_{1}$. From (2.2),

$$
\begin{aligned}
& K_{1}=V^{-1} \Delta_{1 / 2}\left(\frac{1}{2} \log V\right), \\
& K=V \Delta_{1 / 2}\left(-\frac{1}{2} \log V\right) .
\end{aligned}
$$

From (2.5), since $g_{1}$ is spherical, we get $\Delta_{1 / 2} \log V=2 V$ and so from (2.6) $K=$ $-V^{2}$. Therefore

$$
\Delta \log (-K)=2 V \Delta_{1 / 2} \log V=4 V^{2}=-4 K,
$$

hence $g$ is a Ricci metric by Lemma 2.2.

The corresponding statements in positive curvature are similar and left to the reader:

Lemma 2.4. Let $(M, g)$ be a Riemannian surface of positive curvature $K>0$. The following conditions are equivalent:

- $g$ is a Ricci metric;

- $\Delta \log K+4 K=0$;

- the metric $K^{1 / 2} \mathrm{~g}$ is flat;

- the metric Kg is hyperbolic, i.e., of constant Gaussian curvature - 1 .

Lemma 2.5. Let $g_{1 / 2}$ be a flat metric on a surface $M$ and $V \in \mathcal{C}^{\infty}(M), V>0$ such that $g_{1}:=V g_{1 / 2}$ is hyperbolic. Then $g:=V^{-1} g_{1 / 2}$ is a Ricci metric of Gaussian curvature $V^{2}$. 


\section{Spinorial characterization of isometric embeddings in $\mathbb{R}^{3}$}

In [8] Friedrich remarked that local isometric embeddings of a Riemannian surface in the Euclidean space $\mathbb{R}^{3}$ are characterized by special spinor fields on the surface called generalized Killing spinors (see also [2]):

Lemma 3.1 ([8], Theorem 13). Let $W$ be a symmetric tensor on the spin surface $\left(M^{2}, g\right)$. There exists a locally isometric embedding $(M, g) \rightarrow \mathbb{R}^{3}$ with Weingarten tensor $W$ if and only if $M$ carries a non-zero spinor $\psi$ satisfying

$$
\nabla_{X} \psi=\frac{1}{2} W(X) \cdot \psi, \quad(\forall) X \in T M .
$$

Moreover, due to the algebraic structure of spinors in two dimensions, a generalized Killing spinor can be characterized by a seemingly weaker condition:

Lemma 3.2. A non-zero spinor $\psi$ on a Riemannian surface $\left(M^{2}, g\right)$ satisfies (3.1) for some symmetric tensor $W$ if and only if it has constant length and there exists a real function $w$ such that $D \psi=w \psi$. In this situation, $w=-\frac{1}{2} \operatorname{tr}(W)$.

Proof. Assume first that $\psi$ satisfies (3.1). Taking the Clifford contraction in this equation yields $D \psi=-\frac{1}{2} \operatorname{tr}(W) \psi$. Moreover $d|\psi|^{2}(X)=2\left\langle\nabla_{X} \psi, \psi\right\rangle=$ $\langle W(X) \cdot \psi, \psi\rangle=0$ for every tangent vector $X$, so $\psi$ has constant length.

Conversely, assume that $\psi$ has constant length and $D \psi=w \psi$. Since $\psi$ is non-zero, one may assume that $|\psi|=1$. Let $e_{1}, e_{2}$ be a local orthonormal basis of the tangent bundle. For dimensional reasons, the spinors $\psi, e_{1} \cdot \psi, e_{2} \cdot \psi$ and $e_{1} \cdot e_{2} \cdot \psi$ define a local orthonormal basis (over $\mathbb{R}$ ) of the spin bundle. Since $\left\langle\nabla_{X} \psi, \psi\right\rangle=$ $\frac{1}{2} d|\psi|^{2}(X)=0$ for every tangent vector $X$, there exist an endomorphism field $A$ of $T M$ and a 1-form $a$ such that

$$
\nabla_{X} \psi=A(X) \cdot \psi+a(X) e_{1} \cdot e_{2} \cdot \psi
$$

for every $X \in T M$. Let $\left(a_{i j}\right)$ be the matrix of $A$ in the basis $e_{1}, e_{2}$ and $a=$ $a_{1} e_{1}^{*}+a_{2} e_{2}^{*}$. After Clifford contraction, (3.2) yields

$$
w \psi=D \psi=-\left(a_{11}+a_{22}\right) \psi+\left(a_{12}-a_{21}\right) e_{1} \cdot e_{2} \cdot \psi+\left(a_{2} e_{1}-a_{1} e_{2}\right) \cdot \psi .
$$

Using again that $\psi, e_{1} \cdot \psi, e_{2} \cdot \psi$ and $e_{1} \cdot e_{2} \cdot \psi$ are linearly independent over $\mathbb{R}$, we get $w+\operatorname{tr}(A)=0, a_{12}=a_{21}$ and $a=0$. Thus (3.2) is equivalent to (3.1) for $W=2 A$.

Specializing to the case of minimal surfaces, we get:

Corollary 3.3. A Riemannian surface has local isometric minimal embeddings in $\mathbb{R}^{3}$ if and only if it carries local non-zero harmonic spinors of constant length.

This provides a simple characterization of metrics which embed locally as minimal surfaces in $\mathbb{R}^{3}$, in terms of the conformal factor of the metric in isothermal coordinates. 
Corollary 3.4. Let $g_{0}=d x^{2}+d y^{2}$ be the flat metric on some domain $\Omega \subset \mathbb{C}$, and $f: \Omega \rightarrow \mathbb{R}$ any smooth function. The metric $g=e^{-2 f} g_{0}$ has (locally) an isometric embedding in $\mathbb{R}^{3}$ as minimal surface if and only if near every $x \in \Omega$ there exists a pair of holomorphic functions $(a, b)$ such that $e^{-f}=|a|^{2}+|b|^{2}$.

Proof. Let $x \in \Omega$ and assume that some $U \subset \Omega, x \in \mathrm{U}$, has a local isometric embedding as minimal surface in $\mathbb{R}^{3}$. The previous corollary shows the existence of a harmonic spinor $\psi$ of unit length with respect to $g$ defined on some open set $V \subset U, x \in V$. By (2.3), $e^{-f / 2} \psi$ is a harmonic spinor on $\left(V, g_{0}\right)$ of square length $e^{-f}$. The (complex) spin bundle of $\left(\Omega, g_{0}\right)$ is trivial and spanned by two parallel spinors $\psi^{ \pm} \in \mathcal{C}^{\infty}\left(\Sigma^{ \pm} \Omega\right)$. Write $\psi=a \psi^{+}+\bar{b} \psi^{-}$for some complex-valued functions $a, b$ on $V$. Since $D_{0}=\left[\begin{array}{cc}0 & -\partial_{z} \\ \partial_{\bar{z}} & 0\end{array}\right]$ with respect to the basis $\left\{\psi^{+}, \psi^{-}\right\}$, $D_{0} \psi=0$ is equivalent to $a$ and $b$ being holomorphic. The converse statement is similar.

We consider now the case of Riemannian surfaces $\left(M^{2}, g\right)$ (locally) isometrically embedded as space-like surfaces in the Lorentz space $\mathbb{R}^{2,1}$. The restriction of the (complex) spin bundle $\Sigma \mathbb{R}^{2,1}$ to $M$ can be identified with the spin bundle $\Sigma M=\Sigma^{+} M \oplus \Sigma^{-} M$ of $(M, g)$. With respect to this identification, the Clifford action of the time-like normal vector $v$ of square norm -1 is given by $v \cdot \psi=\bar{\psi}:=\psi^{+}-\psi^{-}$and the natural (indefinite) Hermitian product $h$ on $\Sigma \mathbb{R}^{2,1}$ corresponds to $h(\psi, \psi):=\left|\psi^{+}\right|^{2}-\left|\psi^{-}\right|^{2}$ for $\psi=\psi^{+}+\psi^{-}$. The restriction $\psi$ of a parallel spinor from $\Sigma \mathbb{R}^{2,1}$ to $\Sigma M$ satisfies $\nabla_{X} \psi=\frac{1}{2} W(X) \cdot \bar{\psi}$. The arguments from the previous subsection remain valid mutatis mutandis and we obtain the following characterization of maximal embeddings in the Lorentz space:

Lemma 3.5. Let $g_{0}=d x^{2}+d y^{2}$ be the flat metric on some domain $\Omega \subset \mathbb{C}$ and $f: \Omega \rightarrow \mathbb{R}$ any smooth function. The metric $g=e^{-2 f} g_{0}$ admits local isometric embeddings in $\mathbb{R}^{2,1}$ as maximal surface if and only if locally on $\Omega$ there exist pairs of holomorphic functions $(a, b)$ such that $e^{-f}=|a|^{2}-|b|^{2}$.

As a corollary we recover the well-known property that the curvature of minimal surfaces in $\mathbb{R}^{3}$ and of maximal surfaces in $\mathbb{R}^{2,1}$ satisfies the Ricci condition.

Lemma 3.6. Let $\left(M^{2}, g\right) \subset \mathbb{R}^{3}$ be either a minimal surface in $\mathbb{R}^{3}$ or a maximal surface in the Lorentz space $\mathbb{R}^{2,1}$. Then the Gaussian curvature $K$ of $M$ satisfies the Ricci condition (1.1), namely $K \Delta K+g(d K, d K)+4 K^{3}=0$.

Proof. We write $g=e^{-2 f} g_{0}$ where $g_{0}$ is flat. By (2.2) we have

$$
K=-e^{2 f} \Delta_{0} f .
$$

Moreover, Corollary 3.4 and Lemma 3.5 show that $e^{-f}=|a|^{2}+\varepsilon|b|^{2}$ for some holomorphic functions $a, b$. Here the $\operatorname{sign} \varepsilon$ is 1 if we work in $\mathbb{R}^{3}$ and -1 in Lorentz space. The Laplacian $\Delta_{0}$ of the flat metric $g_{0}:=d x^{2}+d y^{2}=|d z|^{2}$ on $\mathbb{R}^{2}$ satisfies

$$
\Delta_{0}=-4 \partial_{z} \partial_{\bar{z}}
$$


where

$$
\partial_{z}:=\frac{\partial}{\partial z}=\frac{1}{2}\left(\frac{\partial}{\partial x}-i \frac{\partial}{\partial y}\right), \quad \partial_{\bar{z}}:=\frac{\partial}{\partial \bar{z}}=\frac{1}{2}\left(\frac{\partial}{\partial x}+i \frac{\partial}{\partial y}\right) .
$$

Using (3.4) we infer

$$
\begin{aligned}
\frac{1}{4} \Delta_{0} f & =-\partial_{z} \partial_{\bar{z}} f=-\partial_{z} \partial_{\bar{z}} \log \left(|a|^{2}+\varepsilon|b|^{2}\right)=\partial_{z}\left(\frac{a \overline{a^{\prime}}+\varepsilon b \overline{b^{\prime}}}{|a|^{2}+\varepsilon|b|^{2}}\right) \\
& =\frac{\left(a^{\prime} \overline{a^{\prime}}+\varepsilon b^{\prime} \overline{b^{\prime}}\right)\left(|a|^{2}+\varepsilon|b|^{2}\right)-\left(a \overline{a^{\prime}}+\varepsilon b \overline{b^{\prime}}\right)\left(a^{\prime} \bar{a}+\varepsilon b^{\prime} \bar{b}\right)}{\left(|a|^{2}+\varepsilon|b|^{2}\right)^{2}} \\
& =\varepsilon \frac{\left|a b^{\prime}-b a^{\prime}\right|^{2}}{\left(|a|^{2}+\varepsilon|b|^{2}\right)^{2}} .
\end{aligned}
$$

We thus obtain $e^{-2 f} \Delta_{0} f=4 \varepsilon\left|a b^{\prime}-b a^{\prime}\right|^{2}$, and since the logarithm of the norm of a non-vanishing holomorphic function is harmonic, we get from (2.1) and (3.3) at points where $K$ does not vanish

$$
\begin{aligned}
0 & =\Delta_{0}\left(\log \left|e^{-2 f} \Delta_{0} f\right|\right)=\Delta_{0}\left(\log \left|e^{2 f} \Delta_{0} f\right|-4 f\right)=\Delta_{0}(\log |K|)-4 \Delta_{0} f \\
& =\Delta_{0}(\log |K|)+4 e^{-2 f} K=e^{-2 f}(\Delta(\log |K|)+4 K)
\end{aligned}
$$

whence $\Delta(\log |K|)+4 K=0$. Using the formula $\Delta=\delta^{g} d$ we obtain

$$
-4 K=\Delta(\log |K|)=\delta^{g}\left(\frac{d K}{K}\right)=\frac{\Delta K}{K}+\frac{g(d K, d K)}{K^{2}},
$$

which is equivalent to (1.1).

Let us remark that there is a close link, already noted by Alías [1], between minimal surfaces in $\mathbb{R}^{3}$ and maximal surfaces in $\mathbb{R}^{2,1}$. In our setting, this duality is obtained by associating to any Ricci metric of the form $\left(|a|^{2}+|b|^{2}\right)^{2}|d z|^{2}$, which by Corollary 3.4 embeds as a minimal surface in $\mathbb{R}^{3}$, the Ricci metric $\left(|a|^{2}-|b|^{2}\right)^{2}|d z|^{2}$ which by Lemma 3.5 embeds as maximal surface in $\mathbb{R}^{2,1}$. This correspondence is not intrinsic since it depends on the choice of the holomorphic functions $a$ and $b$ representing the conformal factor $e^{-f}=|a|^{2}+|b|^{2}$.

\section{Log-harmonic and holomorphic functions}

In this section we prove Theorem 1.3, which is one of the central results of this paper.

Definition 4.1. A real-valued function $F$ defined on some open set $\Omega \subset \mathbb{C}$ is called log-harmonic if $F \in \mathcal{C}^{\infty}(\Omega, \mathbb{R})$ and $\log |F|$ is harmonic on the open set where $F \neq 0$. 
It is clear that for every holomorphic function $h$ on some $\Omega \subset \mathbb{C}$, its square norm $F:=|h|^{2}$ is log-harmonic. Conversely, if $F$ is log-harmonic, and, say, nonnegative, does there exist a holomorphic function $h$ on $\Omega$ such that $F=|h|^{2}$ ? If $F>0$ and $\Omega$ is simply connected, the answer is standard:

Lemma 4.2. Let $F>0$ be a positive log-harmonic function on some simply connected domain $\Omega \subset \mathbb{C}$. Then there exists a holomorphic function $h$ on $\Omega$ such that $F=|h|^{2}$.

Proof. By (3.4) $\partial_{z} \log F$ is holomorphic on $\Omega$ and since $\Omega$ is simply connected, there exists a holomorphic function $g_{1}$ on $\Omega$ with $\partial_{z} \log F=\partial_{z} g_{1}$. Thus $g_{2}:=$ $\overline{\log F-g_{1}}$ is holomorphic on $\Omega$ and $\log F=g_{1}+\overline{g_{2}}$. Since $\log F$ is real, we have $\log F=\Re\left(g_{1}+g_{2}\right)$, so $F=|h|^{2}$ for $h:=e^{\frac{g_{1}+g_{2}}{2}}$.

The question, answered by Theorem 1.3, is whether the local solutions extend globally including on the zero set. We need some preliminary results first.

Lemma 4.3. If two holomorphic non-vanishing functions $h_{1}$ and $h_{2}$ have the same norm on a connected open subset $\Omega \subset \mathbb{C}$ then there exists $\theta \in[0,2 \pi)$ such that $h_{1}=e^{i \theta} h_{2}$ on $\Omega$.

Proof. Clear from the maximum principle applied to $h_{1} / h_{2}$.

For a vector $G=\left(G_{1}, G_{2}\right) \in \mathbb{C}^{2}$ we denote $|G|^{2}:=\left|G_{1}\right|^{2}+\left|G_{2}\right|^{2}$ and $|G|_{-}^{2}:=\left|G_{1}\right|^{2}-\left|G_{2}\right|^{2}$.

Lemma 4.4. Let $\Omega \subset \mathbb{C}$ be a connected domain and $G=\left(G_{1}, G_{2}\right): \Omega \rightarrow$ $\mathbb{C}^{2} \backslash\{(0,0)\}$ be a holomorphic map. If $|G(0)|^{2}>0$ then another holomorphic map $H: \Omega \rightarrow \mathbb{C}^{2}$ satisfies $|G|^{2}=|H|^{2}$ if and only if there exists $A \in \mathrm{U}(2)$ such that $H=A G$. Similarly, if $|G(0)|_{-}^{2}>0$ then another holomorphic map $H: \Omega \rightarrow \mathbb{C}^{2}$ satisfies $|G|_{-}^{2}=|H|_{-}^{2}$ if and only if there exists $A \in \mathrm{U}(1,1)$ such that $H=A G$. Moreover, if $\Delta_{0} \log |G|^{2}$, respectively $\Delta_{0} \log |G|_{-}^{2}$ are not identically 0 , then the matrix $A$ is unique.

Proof. The "if" part is obvious. Assume now that $|G|^{2}=|H|^{2}$. Since $G(0) \neq 0$, one of its components, say $G_{1}$ does not vanish at 0 , and thus on some smaller domain $\Omega^{\prime} \subset \Omega$. The three functions $a:=G_{2} / G_{1}, b:=H_{1} / G_{1}$ and $c:=H_{2} / G_{1}$ are holomorphic on $\Omega^{\prime}$ and satisfy

$$
1+|a|^{2}=|b|^{2}+|c|^{2} \text {. }
$$

Taking the double derivative $\partial_{z} \partial_{\bar{z}}\left(i . e .,-\frac{1}{4} \Delta_{0}\right)$ in this relation yields $\left|a^{\prime}\right|^{2}=\left|b^{\prime}\right|^{2}+$ $\left|c^{\prime}\right|^{2}$. If $a^{\prime} \equiv 0$ on $\Omega^{\prime}$ then $a, b, c$ are constant, hence $G_{2}, H_{1}, H_{2}$ are constant multiples of $G_{1}$ and the conclusion follows from the transitivity of the action of $U(2)$ on the unit sphere $\mathbb{S}^{3}$. Assume that $a^{\prime}$ does not vanish on some disc $\Omega^{\prime \prime} \subset \Omega^{\prime}$. The holomorphic functions $\alpha:=b^{\prime} / a^{\prime}$ and $\beta:=c^{\prime} / a^{\prime}$ satisfy

$$
|\alpha|^{2}+|\beta|^{2}=1
$$


on $\Omega^{\prime \prime}$. Differentiating again with respect to $\partial_{z} \partial_{\bar{z}}$ we get $\left|\alpha^{\prime}\right|^{2}+\left|\beta^{\prime}\right|^{2}=0$, so $\alpha$ and $\beta$ are constant on $\Omega^{\prime \prime}$, and thus on $\Omega^{\prime}$. We then have $b^{\prime}=\alpha a^{\prime}$ and $c^{\prime}=\beta a^{\prime}$ on $\Omega^{\prime}$, so there exist constants $\gamma$ and $\delta$ such that $b=\alpha a+\gamma$ and $c=\beta a+\delta$ on $\Omega^{\prime}$. This reads $H=A G$ on $\Omega^{\prime}$, thus on $\Omega$, where $A=\left[\begin{array}{ll}\gamma & \alpha \\ \delta & \beta\end{array}\right]$. It remains to check that $A \in \mathrm{U}(2)$. From (4.1) we get

$$
1+|a|^{2}=|\alpha a+\gamma|^{2}+|\beta a+\delta|^{2}=|a|^{2}+\left(|\gamma|^{2}+|\delta|^{2}\right)+2 \Re(a(\alpha \bar{\gamma}+\beta \bar{\delta})),
$$

so the imaginary part of the holomorphic function $a(\alpha \bar{\gamma}+\beta \bar{\delta})$ vanishes. Since $a$ is non-constant (see above) we deduce that $\alpha \bar{\gamma}+\beta \bar{\delta}=0$ and $|\gamma|^{2}+|\delta|^{2}=1$. Together with (4.2), this shows that $A \in \mathrm{U}(2)$.

In the semi-definite case the proof proceeds similarly with the same notation: we have $1-|a|^{2}=|b|^{2}-|c|^{2}$ hence $\left|a^{\prime}\right|^{2}=\left|c^{\prime}\right|^{2}-\left|b^{\prime}\right|^{2}$. If $a^{\prime}=0$ then $G_{2}=$ $\gamma G_{1}$ for some constant $\gamma$ and by Lemma $4.3 b^{\prime}=e^{i \theta} c^{\prime}$, which implies easily that $H_{1}, H_{2}$ are constant multiples of $G_{1}$ and the conclusion follows. If $a^{\prime} \neq 0$ the functions $\alpha:=b^{\prime} / a^{\prime}$ and $\beta:=c^{\prime} / a^{\prime}$ satisfy $|\alpha|^{2}-|\beta|^{2}=1$ so $\left|\alpha^{\prime}\right|^{2}-\left|\beta^{\prime}\right|^{2}=0$. These two identities easily imply that $\alpha, \beta$ are constants. The rest of the proof is unchanged.

Now let $F$ be a log-harmonic function. By Lemma 4.2 every point where $F$ is non-zero has an open neighborhood on which there exists a holomorphic function $h$ with $F=|h|^{2}$. The case of isolated zeros is only slightly more involved.

Lemma 4.5. Let $F: \mathbb{D} \rightarrow \mathbb{R}$ be a smooth non-negative function on the unit disc $\mathbb{D} \subset \mathbb{C}$ such that $F$ does not vanish on $\mathbb{D}^{*}:=\mathbb{D} \backslash\{0\}$. If $\log (F)$ is harmonic on $\mathbb{D}^{*}$, then there exists a holomorphic function $h$ on $\mathbb{D}$ such that $F=|h|^{2}$.

Proof. We identify the universal cover $\widetilde{\mathbb{D}^{*}}$ of $\mathbb{D}^{*}$ with $\{z \in \mathbb{C} ; \Re(z)<0\}$ and the projection from $\widetilde{\mathbb{D}^{*}}$ to $\mathbb{D}^{*}$ with the exponential map. The function $z \mapsto \log \left(F\left(e^{z}\right)\right)$ is harmonic on $\widetilde{\mathbb{D}}^{*}$, so by Lemma 4.2 there exists a holomorphic function $G$ on $\mathbb{D}^{*}$ with $F\left(e^{z}\right)=|G(z)|^{2}$ for all $z \in \widetilde{\mathbb{D}^{*}}$. By Lemma 4.3 , there exists $\theta \in[0,1)$ such that $G(z+2 \pi i)=e^{-2 \pi i \theta} G(z)$. The function

$$
H(z):=e^{z \theta} G(z)
$$

is thus invariant by translation with $2 \pi i$, hence it descends to a holomorphic function $h$ on $\mathbb{D}^{*}$ with $h\left(e^{z}\right)=H(z)$. Denoting $w:=e^{z}$ we get

$$
F(w)=|G(z)|^{2}=\left|e^{-2 z \theta} H(z)\right|^{2}=|w|^{-2 \theta}|h(w)|^{2} .
$$

This shows in particular that the function $h$ is bounded near the origin, so it extends to a holomorphic function $h$ on $\mathbb{D}$.

Let $k$ be the vanishing order of $h$ at 0 . One has $h(w)=h_{1}(w) w^{k}$ with $h_{1}$ holomorphic and $h_{1}(0) \neq 0$. Since $w \mapsto|w|^{2 k-2 \theta}=F /\left|h_{1}\right|^{2}$ is smooth near 0 , the exponent $2 k-2 \theta$ is an even integer. By the choice of $\theta$ in $[0,1)$ we get $\theta=0$, so $F=|h|^{2}$ as claimed. 
The main difficulty in Theorem 1.3 is to show that a log-harmonic function does not have non-isolated zeros.

Theorem 4.6. Let $F: \mathbb{D} \rightarrow \mathbb{R}$ be a smooth function on the unit disc $\mathbb{D} \subset \mathbb{C}$ such that $\log (|F|)$ is harmonic on $\mathbb{D} \backslash F^{-1}(\{0\})$. Then either $F$ vanishes identically, or $F^{-1}(\{0\})$ is a discrete set. In particular, log-harmonic functions on $\mathbb{D}$ have constant sign.

Proof. The proof will be divided in several steps.

Lemma 4.7. Let $z_{0} \in \mathbb{D}$ be a non-isolated zero of a log-harmonic function $F$. Then $F$ vanishes at infinite order at $z_{0}$.

Proof. On the open set $\mathbb{D} \backslash F^{-1}(\{0\})$ the function $\log (|F|)$ is harmonic, thus

$$
0=\Delta_{0}(\log (|F|))=\delta^{0}(d F / F)=\Delta_{0}(F) / F+|d F|^{2} / F^{2},
$$

therefore

$$
F \Delta_{0}(F)+|d F|^{2}=0 .
$$

By restricting to a small disc centered at $z_{0}$ and composing $F$ with a translation one may take $z_{0}=0$. Assume that $F$ does not vanish at infinite order at 0 and let $P$ be the principal part of $F$ near 0 . Then $P$ is a homogeneous polynomial in $x, y$ such that $F-P=o\left(r^{n}\right)$, where $r:=\sqrt{x^{2}+y^{2}}$ and $n$ is the degree of $P$. Clearly $\Delta_{0}(F)=\Delta_{0}(P)+o\left(r^{n-2}\right)$ and $d F=d P+o\left(r^{n-1}\right)$. From (4.3) we get $P \Delta_{0}(P)+|d P|^{2}=o\left(r^{2 n-2}\right)$. On the other hand the left-hand side in this equality is a homogeneous polynomial in $x, y$ of degree $2 n-2$, thus showing that

$$
P \Delta_{0}(P)+|d P|^{2}=0 \text {. }
$$

In polar coordinates we can write $P=r^{n} Q(\theta)$, where $Q(\theta)=P(\cos \theta, \sin \theta)$ is a trigonometric polynomial with real coefficients. Using the formulae

$$
d x^{2}+d y^{2}=d r^{2}+r^{2} d \theta^{2}, \quad \Delta_{0}=-\left(\frac{1}{r} \frac{\partial}{\partial r}+\frac{\partial^{2}}{\partial r^{2}}+\frac{1}{r^{2}} \frac{\partial^{2}}{\partial \theta^{2}}\right),
$$

equation (4.4) becomes

$$
-r^{n} Q\left[n r^{n-2} Q+n(n-1) r^{n-2} Q+r^{n-2} Q^{\prime \prime}\right]+n^{2} r^{2 n-2} Q^{2}+r^{2 n-2} Q^{\prime 2}=0
$$

i.e., $Q^{\prime 2}=Q Q^{\prime \prime}$. The solutions of this differential equation are $Q(\theta)=a e^{b \theta}$ for $a, b \in \mathbb{R}$. Since $Q$ is a trigonometric polynomial, we necessarily have $b=0$ and thus $Q$ is constant. Therefore $P(x, y)=a\left(x^{2}+y^{2}\right)^{n / 2}$ and $a \neq 0$ by the assumption that $P \neq 0$. Incidentally this implies that $n$ is even, but we do not need this observation. More importantly, since $F(z)=P(z)+o\left(|z|^{n}\right)=|z|^{n}(a+o(1))$, it turns out that 0 is an isolated zero of $F$, contradicting the hypothesis. This proves the lemma. 
Let $Z$ denote the (closed) set of non-isolated zeros of $F$. Assume that $F$ does not vanish identically on $\mathbb{D}$ and let $E$ denote a connected component of the open set $\mathbb{D} \backslash Z$. By changing the sign of $F$ if necessary, we can assume that $F$ is non-negative on $E$.

If $E$ is simply connected, by Lemma 4.2 we can construct a holomorphic function $h$ on $E$ such that $F=|h|^{2}$. Since by Lemma $4.7 F$ must vanish to infinite order at every point of $Z$, the function $F \chi_{E}$ is smooth on $\mathbb{D}$, where $\chi_{E}$ is the characteristic function of $E$. Moreover, for every $z_{0} \in \mathbb{D} \backslash E$ we have $F(z) \chi_{E}(z)=o\left(\left|z-z_{0}\right|\right)$.

Extend the holomorphic function $h^{2}$ from $E$ to $\mathbb{D}$ by setting it to be 0 on $\mathbb{D} \backslash E$. At a point $z_{0} \in \mathbb{D} \backslash E$ we have

$$
\frac{\left|h^{2}(z)-h^{2}\left(z_{0}\right)\right|}{\left|z-z_{0}\right|}=\frac{F(z) \chi_{E}(z)}{\left|z-z_{0}\right|}
$$

tends to 0 as $z \rightarrow z_{0}$. Therefore $h^{2}$ is holomorphic on $\mathbb{D}$, and thus its zeros are isolated, which is the conclusion of Theorem 4.6.

We are left with the case where there are no simply connected components of $\mathbb{D} \backslash Z$. Thus, we may assume that $E$ is not simply connected, hence we can find a smooth simple curve $C$ in $E$ containing at least one non-isolated zero of $F$ in its interior. By slightly deforming $C$ if necessary, we can assume that $C$ avoids also the isolated zeros of $F$, i.e., $F$ does not vanish on $C$. Using the Riemann uniformization theorem, we can identify the interior of $C$ with the unit disk $\mathbb{D}$. We can thus from now on assume that $F: \overline{\mathbb{D}} \rightarrow \mathbb{R}$ is smooth, non-negative, has at least one nonisolated zero, is log-harmonic outside its zero-set, and does not vanish on $\mathbb{S}^{1}$.

Using the solution to the Dirichlet problem, we find a harmonic function $\phi$ : $\mathbb{D} \rightarrow \mathbb{R}$ such that $\phi=\log (F)$ on $\mathbb{S}^{1}$. Replacing $F$ with $e^{-\phi} F$ (whose logarithm is clearly harmonic outside its zero set), we can thus assume that $F$ equals 1 on $\mathbb{S}^{1}$.

We now recall that for every harmonic function defined on an annulus $C\left(r_{1}, r_{2}\right):=$ $\left\{z\left|r_{1} \leq\right| z \mid \leq r_{2}\right\}$, its mean values along the concentric circles $|z|=r$ have a special behavior.

Lemma 4.8. Assume that $f: C\left(r_{1}, r_{2}\right) \rightarrow \mathbb{R}$ is harmonic. Then there exist real constants $a, b$ such that

$$
\int_{C(r)} f d l=r(\mu \log (r)+v)
$$

for every $r \in\left[r_{1}, r_{2}\right]$, where dl denotes the length element. We call $\mu$ the virtual measure of $f$ and denote it by $\mu(f)$. If $f$ extends to a harmonic function on the disk $\left\{|z| \leq r_{2}\right\}$, then its virtual measure vanishes.

Proof. Let us set

$$
K(r):=r^{-1} \int_{C(r)} f d l=\int_{0}^{2 \pi} f(r \cos t, r \sin t) d t .
$$


Then

$$
\begin{aligned}
K^{\prime}(r) & :=\int_{0}^{2 \pi}\left[\partial_{x} f(r \cos t, r \sin t) \cos t+\partial_{y} f(r \cos t, r \sin t) \sin t\right] d t \\
& =r^{-1} \int_{C(r)} \partial_{x} f d y-\partial_{y} f d x .
\end{aligned}
$$

Using this and the Green-Riemann theorem on $C\left(r_{1}, r_{2}\right)$ we get

$$
\begin{aligned}
0 & =\int_{C\left(r_{1}, r_{2}\right)} \Delta_{0}(f) d x d y=\int_{C\left(r_{2}\right)} \frac{\partial f}{\partial y} d x-\frac{\partial f}{\partial x} d y-\int_{C\left(r_{1}\right)} \frac{\partial f}{\partial y} d x-\frac{\partial f}{\partial x} d y \\
& =r_{1} K^{\prime}\left(r_{1}\right)-r_{2} K^{\prime}\left(r_{2}\right) .
\end{aligned}
$$

This shows that there exists a constant $\mu$ such that $r K^{\prime}(r)=\mu$, thus proving the first claim.

If $f$ is defined on the whole disk, then $K(r)$ is bounded as $r$ tends to 0 , so necessarily $\mu(f)=0$.

Notice that the virtual measure defined in Lemma 4.8 is additive: $\mu\left(f_{1}+f_{2}\right)=$ $\mu\left(f_{1}\right)+\mu\left(f_{2}\right)$.

Returning to our $\log$-harmonic function $F$ and setting $f:=\log (F)$, we shall exploit the fact that $f$ is harmonic on some annulus $C\left(r_{1}, 1\right)$ and vanishes on the outer circle $C(1)$.

Lemma 4.9. Let $F: \overline{\mathbb{D}} \rightarrow[0, \infty)$ be a smooth log-harmonic function with at least one non-isolated zero in $\mathbb{D}$ and identically equal to 1 on $\mathbb{S}^{1}$. Then the virtual measure of $f=\log (F)$ is positive.

Proof. We apply (4.3) and the Green-Riemann formula on the disk $\mathbb{D}$ to get

$0<2 \int_{\mathbb{D}}|d F|^{2} d x \wedge d y=\int_{\mathbb{D}}\left(|d F|^{2}-F \Delta_{0}(F)\right) d x \wedge d y=\int_{\mathbb{S}^{1}} F \frac{\partial F}{\partial x} d y-F \frac{\partial F}{\partial y} d x$.

Using (4.5) and the fact that $F \equiv 1$ on $\mathbb{S}^{1}$, the right-hand term reads

$$
\int_{\mathbb{S}^{1}} F \frac{\partial F}{\partial x} d y-F \frac{\partial F}{\partial y} d x=\int_{\mathbb{S}^{1}} \frac{\partial f}{\partial x} d y-\frac{\partial f}{\partial y} d x=\mu(f),
$$

so the virtual measure of $f$ is positive.

Let $z_{0} \in \mathbb{D}$ be a non-isolated zero of $F$. By composing with an element of $\operatorname{Aut}(\mathbb{D})$ if necessary, we can assume $z_{0}=0$. The virtual measure of the function $\log |z|$ is by direct computation equal to $2 \pi$. For every positive integer $n$, the function $F_{n}(z):=|z|^{-n} F(z)$ is smooth by Lemma 4.7. The $\operatorname{logarithm} f_{n}:=\log \left(F_{n}\right)=$ $f-n \log |z|$ is clearly harmonic on its domain of definition, and the restriction of $f_{n}$ to $\mathbb{S}^{1}$ vanishes. We can thus apply Lemma 4.9 to $f_{n}$ and deduce that $\mu\left(f_{n}\right)>0$. On the other hand the virtual measure is additive, so $\mu\left(f_{n}\right)=\mu(f)-2 \pi n$ is negative for $n$ large enough. This contradiction shows that $F$ does not have any non-isolated zeros, and proves the theorem. 
Proof of Theorem 1.3. By Lemma 4.5 and Theorem 4.6, for every $\alpha \in \Omega$ there exists an open disk $U_{\alpha} \ni \alpha$ and a holomorphic function $h_{\alpha}: U_{\alpha} \rightarrow \mathbb{C}$ with $|F|=$ $\left|h_{\alpha}\right|^{2}$. Lemma 4.3 shows that for every $\alpha$ and $\beta$ there exists a unique $A_{\alpha \beta} \in \mathbb{S}^{1}$ with $h_{\alpha}=A_{\alpha \beta} h_{\beta}$ on $U_{\alpha} \cap U_{\beta}$. The Čech cocycle $\left(A_{\alpha \beta}\right)$ must be exact since $\pi_{1}(\Omega)=1$. Thus $A_{\alpha \beta}=A_{\alpha}^{-1} A_{\beta}$ for some $A_{\alpha} \in \mathbb{S}^{1}$, and so $A_{\alpha} h_{\alpha}$ agree on intersections, thus defining a global solution $h$ on $\Omega$ satisfying $|F|=|h|^{2}$.

\section{Local embedding of Ricci metrics}

This section is devoted to the following:

Proof of Theorem 1.2. Every point in $M$ has a neighborhood where the metric $g$ can be written as $g=e^{-2 f} g_{0}$, where $g_{0}$ is flat and $f$ is smooth. By (2.1) and (2.2), $\Delta f=-K$. By Lemma 2.2, the Ricci condition (1.1) implies $\Delta\left(\log \left|e^{-4 f} K\right|\right)=0$ at points where $K$ does not vanish, in other words $e^{-4 f} K$ is log-harmonic. Theorem 4.6 implies that if $K$ does not vanish identically, then it has only isolated zeros and does not change sign on $M$.

Case 1: $K \leq 0$ on $M$. Let $P$ be an arbitrary point of $M$. Choose a neighborhood $\mathbb{D} \ni P$ such that $K<0$ on $\mathbb{D} \backslash\{P\}$. We can identify $\left(\mathbb{D}, g_{0}\right)$ with a disk in $\mathbb{C}$ endowed with the Euclidean metric $|d z|^{2}$ so that $P$ corresponds to 0 .

Case 1.1: $K \leq 0, K(0) \neq 0$. This was originally treated by Ricci-Curbastro [18], we give here an argument in our framework. By Lemma 2.2, the Ricci condition (1.1) implies that the metric $g_{r}:=(-K)^{r} g$ is flat for $r=\frac{1}{2}$ and has constant Gaussian curvature equal to 1 for $r=1$. Consequently, by shrinking $\mathbb{D}$ if necessary, we may assume that there exist isometries

$$
\varphi:\left(\mathbb{D}, g_{1 / 2}\right) \rightarrow\left(U_{0},|d z|^{2}\right), \quad U_{0} \subset \mathbb{C}
$$

and

$$
\psi:\left(\mathbb{D}, g_{1}\right) \rightarrow\left(U_{1}, \frac{4|d z|^{2}}{\left(1+|z|^{2}\right)^{2}}\right), \quad U_{1} \subset \mathbb{C}
$$

The maps $\varphi$ and $\psi$ are holomorphic functions of $z$, so we can write

$$
g_{1 / 2}=\sqrt{-K} e^{-2 f}|d z|^{2}=\left|\varphi^{\prime}\right|^{2}|d z|^{2}, \quad g_{1}=(-K) e^{-2 f}|d z|^{2}=\frac{4\left|\psi^{\prime}\right|^{2}|d z|^{2}}{\left(1+|\psi|^{2}\right)^{2}}
$$

whence

$$
e^{-f}=\left(1+|\psi|^{2}\right) \frac{\left|\varphi^{\prime}\right|^{2}}{2\left|\psi^{\prime}\right|}
$$


Since $\psi^{\prime}$ does not vanish on $\mathbb{D}$, there exists a holomorphic map $\zeta: \mathbb{D} \rightarrow \mathbb{C}$ with $\zeta^{2}=2 \psi^{\prime}$. Thus $e^{-f}=|a|^{2}+|b|^{2}$ for holomorphic functions $a:=\frac{\varphi^{\prime}}{\zeta}$ and $b:=\frac{\psi \varphi^{\prime}}{\zeta}$, so by Corollary $3.4,(\mathbb{D}, g)$ has an isometric minimal embedding in $\mathbb{R}^{3}$.

Case 1.2: $K \leq 0, K(0)=0$. Using Case 1.1 treated above, for every point $\alpha \in \mathbb{D} \backslash\{0\}$ there exists an open disk $U_{\alpha} \subset \mathbb{D} \backslash\{0\}$ containing $\alpha$ and a holomorphic function $g_{\alpha}: U_{\alpha} \rightarrow \mathbb{C}^{2} \backslash\{0\}$ such that $e^{-f}=\left|g_{\alpha}\right|^{2}$ on $U_{\alpha}$. Moreover, since $K$ does not vanish on $U_{\alpha}$, we have $\Delta_{0} \log \left(\left|g_{\alpha}\right|^{2}\right) \neq 0$. By Lemma 4.4, there exist unique matrices $A_{\alpha \beta} \in \mathrm{U}(2)$ with $g_{\alpha}=A_{\alpha \beta} g_{\beta}$ on $U_{\alpha} \cap U_{\beta}$, which clearly form a Čech cocycle.

Consider the universal cover $\widetilde{\mathbb{D}^{*}}=\{z \in \mathbb{C} ; \mathfrak{R}(z)<0\}$ of $\mathbb{D}^{*}$ and the projection $p: \widetilde{\mathbb{D}^{*}} \rightarrow \mathbb{D}^{*}$ given by the exponential map. We denote $V_{\alpha}:=p^{-1}\left(U_{\alpha}\right)$ and $G_{\alpha}(z):=g_{\alpha}\left(e^{z}\right)$. Since $\check{H}^{1}\left(\widetilde{\mathbb{D}}^{*} ; \mathrm{U}(2)\right)=0$, the Čech cocycle $\left(V_{\alpha}, A_{\alpha \beta}\right)$ is exact, so there exist locally constant functions $A_{\alpha}: V_{\alpha} \rightarrow \mathrm{U}(2)$ with $A_{\alpha \beta}=A_{\alpha}^{-1} A_{\beta}$ on $V_{\alpha} \cap V_{\beta}$. This shows the existence of a global holomorphic map $G: \widetilde{\mathbb{D}}^{*} \rightarrow \mathbb{C}^{2}$ (given by $G=A_{\alpha} G_{\alpha}$ on $V_{\alpha}$ ), with $e^{-f\left(e^{z}\right)}=|G(z)|^{2}$ for all $z \in \widetilde{\mathbb{D}^{*}}$. By Lemma 4.3, there exists $A \in \mathrm{U}(2)$ such that $G(z+2 \pi i)=A G(z)$. We diagonalize $A=$ $P\left[\begin{array}{cc}e^{2 \pi i \theta_{1}} & 0 \\ 0 & e^{2 \pi i \theta_{2}}\end{array}\right] P^{-1}$ for $P \in \mathrm{U}(2), \theta_{1}, \theta_{2} \in[0,1)$.

The map

$$
H(z):=\left[\begin{array}{cc}
e^{-z \theta_{1}} & 0 \\
0 & e^{-z \theta_{2}}
\end{array}\right] P^{-1} G(z)
$$

is invariant by translation with $2 \pi i$, hence it descends to a holomorphic map $h=$ $\left(h_{1}, h_{2}\right): \mathbb{D}^{*} \rightarrow \mathbb{C}^{2}$ with $h\left(e^{z}\right)=H(z)$. Setting $w:=e^{z}$ we get

$$
e^{-f(w)}=|G(z)|^{2}=\left|\left[\begin{array}{cc}
e^{z \theta_{1}} & 0 \\
0 & e^{z \theta_{2}}
\end{array}\right] h(w)\right|^{2}=\left|h_{1}(w)\right|^{2}|w|^{2 \theta_{1}}+\left|h_{2}(w)\right|^{2}|w|^{2 \theta_{2}} .
$$

Let $k_{j}$ be the vanishing order of $h_{j}$ at 0 . One has $h_{j}(w)=l_{j}(w) w^{k_{j}}$ with $l_{j}$ holomorphic and $l_{j}(0) \neq 0$. We thus have

$$
e^{-f(w)}=l_{1}(w)|w|^{r_{1}}+l_{2}(w)|w|^{r_{2}}
$$

where $l_{i}$ are smooth functions which do not vanish near 0 and $r_{j}=2 k_{j}+2 \theta_{j}$. Such a function is smooth if and only if $r_{1}$ and $r_{2}$ are both even integers, which implies $\theta_{j}=0$, so $e^{-f}=|h|^{2}$ and the conclusion follows from Corollary 3.4.

Case 2: $K \geq 0$ on $M$.

Case 2.1: $K \geq 0, K(0) \neq 0$. For $r \in \mathbb{R}$ let $g_{r}:=K^{r} g$. Then as in Case 1.1, using Lemma 2.4 we find holomorphic maps $\varphi, \psi$ from $\mathbb{D}^{\prime} \subset \mathbb{D}$ to $\mathbb{C}$, respectively to $\mathbb{H}^{2}=\mathbb{D}$, satisfying

$$
\left\{\begin{array}{l}
g_{1 / 2}:=\sqrt{K} e^{-2 f}|d z|^{2}=\left|\varphi^{\prime}\right|^{2}|d z|^{2} \\
g_{1}:=K e^{-2 f}|d z|^{2}=\frac{4\left|\psi^{\prime}\right|^{2}|d z|^{2}}{\left(1-|\psi|^{2}\right)^{2}}
\end{array}\right.
$$


It follows that $e^{-f}=|a|^{2}-|b|^{2}$ for holomorphic functions $a:=\frac{\varphi^{\prime}}{\zeta}$ and $b:=\frac{\psi \varphi^{\prime}}{\zeta}$, for some square root $\zeta$ of $2 \psi^{\prime}$. By Lemma 3.5, a neighborhood of 0 in the disk $\left(\mathbb{D}^{\prime}, g\right)$ has an isometric maximal embedding in $\mathbb{R}^{2,1}$.

Case 2.2: $K \geq 0, K(0)=0$. The proof is more involved than in Case 1.2, essentially because the group $\mathrm{U}(1,1)$ of isometries of the indefinite Hermitian form $|\cdot|-$ is non-compact. Using Case 2.1 we obtain like before a holomorphic map $G: \widetilde{\mathbb{D}^{*}} \rightarrow \mathbb{C}^{2}$ with $|G(z)|_{-}^{2}=e^{-f\left(e^{z}\right)}$. By the second part of Lemma 4.4, $G(z+$ $2 \pi i)=A G(z)$ for some matrix $A \in \mathrm{U}(1,1)$. We wish to show that $A=1$, and then that $G$ descends to a map from $\mathbb{D}^{*} \rightarrow \mathbb{C}^{2}$ which extends holomorphically to $\mathbb{D}$.

Every element $A$ of $\mathrm{U}(1,1)$ is conjugated (inside $\mathrm{U}(1,1))$ to a matrix of the form $e^{2 \pi i \theta} B$ with $\theta \in[0,1)$ and $B$ one of

$$
\begin{array}{ll}
A_{1}=\left[\begin{array}{cc}
e^{2 \pi i \alpha} & 0 \\
0 & e^{-2 \pi i \alpha}
\end{array}\right], & A_{2}=\left[\begin{array}{cc}
1+2 \pi i a & 2 \pi a \\
2 \pi a & 1-2 \pi i a
\end{array}\right], \\
A_{3} & =\left[\begin{array}{cc}
\cosh (2 \pi t) & -\sinh (2 \pi t) \\
-\sinh (2 \pi t) & \cosh (2 \pi t)
\end{array}\right]
\end{array}
$$

for some real constants $\theta, \alpha, a, t$. The three cases occur according to whether $|\operatorname{tr}(A)|$ is smaller, equal or larger than 2 . Consider the group morphisms $B_{j}:(\mathbb{C},+) \rightarrow$ $\mathrm{GL}_{2}(\mathbb{C})$ defined by

$$
\begin{array}{ll}
B_{1}(z)=\left[\begin{array}{cc}
e^{z \alpha} & 0 \\
0 & e^{-z \alpha}
\end{array}\right], & B_{2}(z)=\left[\begin{array}{cc}
1+z a & z a \\
z a & 1-z a
\end{array}\right], \\
B_{3}(z)=\left[\begin{array}{ll}
\cosh (i z t) & \sinh (i z t) \\
\sinh (i z t) & \cosh (i z t)
\end{array}\right] . &
\end{array}
$$

We clearly have $B_{j}(2 \pi i)=A_{j}, j=1,2,3$. It follows that, if $A=P e^{2 \pi i \theta} A_{j} P^{-1}$, then

$$
H(z):=e^{-z \theta} B_{j}(z)^{-1} P G(z)
$$

is invariant by the translation with $2 \pi i$, hence it descends to a map $h: \mathbb{D}^{*} \rightarrow \mathbb{C}^{2}$ satisfying $h\left(e^{z}\right)=H(z)$. Since $B_{j}$ depends holomorphically on $z$, the map $h$ is also holomorphic. Let $w=e^{z}, z=x+i y$ and $r:=|w|$. Denoting $B_{j}=\left(b_{k l}\right)_{1 \leq k, 1 \leq 2}$ we have

$$
\begin{aligned}
e^{-f(w)}=|G(z)|_{-}^{2}=\left|e^{z \theta} B_{j}(z) h(w)\right|_{-}^{2}=r^{2 \theta}\left|B_{j}(z) h(w)\right|_{-}^{2} \\
=r^{2 \theta}\left(\left(\left|b_{11}\right|^{2}-\left|b_{12}\right|^{2}\right)\left|h_{1}\right|^{2}-\left(\left|b_{22}\right|^{2}-\left|b_{21}\right|^{2}\right)\left|h_{2}\right|^{2}\right. \\
\left.\quad+2 \Re\left(\left(b_{11} \bar{b}_{12}-b_{21} \bar{b}_{22}\right) h_{1} \bar{h}_{2}\right)\right) .
\end{aligned}
$$

In each of the three cases we compute

$$
r^{-2 \theta} e^{-f(w)}= \begin{cases}r^{2 \alpha}\left|h_{1}\right|^{2}-r^{-2 \alpha}\left|h_{2}\right|^{2}, & j=1, \\ \left|h_{1}\right|^{2}(1+2 a \log r)+\left|h_{2}\right|^{2}(-1+2 a \log r)+2 \Im\left(h_{1} \bar{h}_{2}\right) a y, & j=2, \\ \left(|h|_{-}^{2} \cos (2 t \log r)+2 \Im\left(h_{1} \bar{h}_{2}\right) \sin (2 t \log r)\right), & j=3 .\end{cases}
$$


For $j=1$ it is clear from the Picard theorem that $h_{1}$ cannot have a essential singularity at 0 , and then the same reasoning applies to $h_{2}$ to deduce that $h$ is meromorphic in 0 . Then since $e^{-f}$ is smooth, it follows that $\alpha \in \mathbb{Z}$, hence $A=e^{2 \pi i \theta} I_{2}$.

For $j=2$, the right-hand side must be $2 \pi$-periodic in $y$ so if $a \neq 0$ then $h_{1}, h_{2}$ are proportional, which would imply that the curvature vanishes identically. Hence $a=0$ and so $A=I_{2}$.

For $j=3$ take $r_{k}=e^{-k \pi / t}$ for $k \in \mathbb{N}$. On the circles of radii $r_{k} \rightarrow 0$, the function $\left|h_{1}\right|$ is uniformly bounded from below since $\theta \geq 0$. By the maximum principle, it must be bounded from below in a neighborhood of 0 and hence $h_{1}$ is meromorphic in 0. Picard's theorem again shows that $h_{2}$ is meromorphic at 0 . With a little more effort one sees that $t$ must be 0 .

In all three cases we have obtained $A=e^{2 \pi i \theta} I_{2}$ and $h$ meromorphic at 0 . In order for $r^{2 \theta}|h|_{-}^{2}$ to be smooth, it is necessary that $\theta=0$ (we cannot have $|h|_{-}^{2}=0$ since this would entail the vanishing of the Gaussian curvature $K$ ). Then clearly $h$ is holomorphic at 0 , so Lemma 3.5 ends the proof.

\section{Compact Ricci surfaces}

In this section we study compact Ricci surfaces without boundary. From Theorem 1.2 , for any such surface, the Gaussian curvature $K$ does not change sign on $M$, so integrating (1.1) over $M$ we see that $K$ has to be non-positive. In the non-negative curvature case we enlarge therefore the class of compact Ricci surfaces by allowing conical singularities. Our examples of compact Ricci surfaces stem from three main sources: triply periodic surfaces, branched coverings of $\mathbb{S}^{2}$, and spherical manifolds with conical singularities.

\subsection{Triply periodic minimal surfaces}

A complete minimal surface $S \subset \mathbb{R}^{3}$ is called triply periodic if it is invariant under the translation group defined by a lattice $\Lambda \subset \mathbb{R}^{3}$. By Lemma 3.6, the quotient $M:=S / \Lambda$ is a compact Ricci surface.

Triply periodic minimal surfaces in $\mathbb{R}^{3}$ are abundant in the literature. The first five examples were constructed by Schwarz at the end of the $19^{\text {th }}$ century. Later on, in his 1970 NASA technical report [20] (see also [11]), Schoen constructed 17 new examples of such surfaces. A significant number of papers appeared since then on this subject, a partial account of which can be found in [15]. Recently Traizet [22] proved that for every lattice $\Lambda \subset \mathbb{R}^{3}$ and for every $g \geq 3$, there exists a minimal surface $S$ in $\mathbb{R}^{3}$ invariant by $\Lambda$ such that $S / \Lambda$ has genus $g$. In particular, this shows the existence of compact Ricci surfaces in any genus $g \geq 3$.

Recall now that for every minimal surface $S \subset \mathbb{R}^{3}$, the Gauss map $G: S \rightarrow \mathbb{S}^{2}$ is a branched covering whose branching points are precisely the zeros of the Gaussian curvature of $S$ (see [15, Proposition 2.1 and Corollary 2.1]). Consequently, if $S$ is triply periodic, the compact Ricci surface $M:=S / \Lambda$ is a branched covering of $\mathbb{S}^{2}$ too. 
Note that the compact Ricci surfaces obtained in this way are branched coverings of $\mathbb{S}^{2}$ with $n$ sheets and have genus $g=n+1 \geq 3$ ([15, Theorem 3.1]).

\subsection{Spherical surfaces with conical singularities}

We have seen in Lemma 2.2 that the metric $g_{1}:=(-K) g$ is locally isometric to $\mathbb{S}^{2}$ and the metric $g_{1 / 2}:=\sqrt{-K} g$ is flat for every Ricci surface $(M, g)$ with nonpositive Gaussian curvature $K$. Of course, the metrics $g_{1 / 2}$ and $g_{1}$ have (conical) singularities at points where $K$ vanishes. This suggests the idea of constructing a flat metric $g_{1 / 2}$ with conical singularities on a given Riemann surface $M$, then a spherical metric $g_{1}=V g_{1 / 2}$ with conical singularities in the same conformal class, and then use Lemma 2.3 to show that the metric $g:=V^{-1} g_{1 / 2}$ is a Ricci metric.

Lemma 6.1. Let $(M, J)$ be a Riemann surface, $\mathcal{P} \subset M$ a discrete set and $\beta: \mathcal{P} \rightarrow$ $\mathbb{R}$ a function. In case $M$ is closed, assume that

$$
\sum_{P \in \mathcal{P}}(\beta(P)-2 \pi)=-2 \pi \chi(M)
$$

Let $z$ be a complex coordinate on $M$ near $\mathcal{P}$. Then there exists a flat metric $g$ on $M \backslash \mathcal{P}$ compatible with $J$ which near each $P \in \mathcal{P}$ is of the form

$$
g=e^{2 v}|z|^{\frac{\beta(P)}{\pi}-2}|d z|^{2}
$$

for some $v \in \mathcal{C}^{\infty}(M, \mathbb{R})$.

Proof. Consider a metric $h$ in the conformal class of $M$ (i.e., compatible with $J$ ), such that $h=|d z|^{2}$ near $\mathcal{P}$. Let $u$ be a smooth positive function on $M \backslash \mathcal{P}$ which equals $|z|^{\frac{\beta\left(P_{j}\right)}{\pi}-2}$ near every $P_{j} \in \mathcal{P}$. Since $\Delta_{h} \log u$ vanishes near $\mathcal{P}$, it extends to a smooth function on $M$. We try to solve the Laplace equation

$$
\Delta_{h} v+K_{h}+\frac{1}{2} \Delta_{h} \log u=0
$$

with $v \in \mathcal{C}^{\infty}(M, \mathbb{R})$. Like every elliptic equation with the unique continuation property, (6.1) can be solved inside $\mathcal{C}^{\infty}$ functions on any non-compact manifold [14, Theorem 5, page 341]. When $M$ is a closed surface, the equation $\Delta v=H$ has solutions if and only if $H$ has zero mean. The set $\mathcal{P}$ is finite, $\mathcal{P}=\left\{P_{1}, \ldots, P_{k}\right\}$. By Gauss-Bonnet and [16, Lemma 4], the integral of $K_{h}+\frac{1}{2} \Delta_{h} \log u$ equals

$$
\int_{M}\left(K_{h}+\frac{1}{2} \Delta_{h} \log u\right) \operatorname{vol}_{h}=2 \pi \chi(M)+\pi \sum_{j=1}^{k}\left(\frac{\beta\left(P_{j}\right)}{\pi}-2\right),
$$

which vanishes precisely when (6.3) holds. Let therefore $v$ be a solution to (6.1). From (2.2), the metric $g:=e^{2 v} u h$ is flat, and by construction near each $P_{j} \in \mathcal{P}$ it takes the desired form. 
The above result is due to Troyanov [23] in the case where $M$ is closed, see also [16].

We define a conical spherical metric on $M$ to be a metric $g_{1}$ of curvature 1 outside an isolated set $\mathcal{P}$ which in some holomorphic coordinate $z$ near each $P_{j} \in \mathcal{P}$ takes the form

$$
g_{1}=\frac{4 n_{j}^{2}|z|^{2 n_{j}-2}|d z|^{2}}{\left(1+|z|^{2 n_{j}}\right)^{2}}
$$

for some $n_{j} \in(0, \infty)$. The number $\alpha_{j}=2 \pi n_{j}$ is the cone angle at $P_{j}$. This definition makes sense for real $n_{j}$ but for us it will be useful for $n_{j} \in \mathbb{N}^{*}$.

Proposition 6.2. Let $\left(M, g_{1}\right)$ be a Riemannian surface with a spherical metric with conical singularities of angles $\alpha_{j}=2 \pi n_{j}$ with $n_{j} \geq 2, n_{j} \in \mathbb{Z}$ at each $P_{j} \in \mathcal{P} \subset$ $M$. If $M$ is closed, assume additionally that the conical angles $\alpha_{1}, \ldots, \alpha_{k}$ at the conical points $P_{1}, \ldots, P_{k}$ satisfy

$$
\sum_{j=1}^{k}\left(\alpha_{j}-2 \pi\right)=-4 \pi \chi(M)
$$

where $\chi(M)$ is the Euler characteristic of $M$. Then $M$ admits a Ricci metric in the conformal class of $g_{1}$.

Proof. Near every conical point of angle $\alpha_{j}$, there exists a complex parameter $z$ with respect to which the spherical metric takes the form (6.2). The function $P_{j} \mapsto$ $\beta\left(P_{j}\right):=\frac{\alpha_{j}}{2}+\pi$ satisfies the hypothesis of Lemma 6.1 if and only if (6.3) holds. From Lemma 6.1, there exists on $M \backslash \mathcal{P}$ a flat metric $g_{1 / 2}$ conformal to $g_{1}$ which near $P_{j} \in \mathcal{P}$ is of the form

$$
g_{1 / 2}=e^{2 v}|z|^{n_{j}-1}|d z|^{2}
$$

for some smooth $v \in \mathcal{C}^{\infty}(M, \mathbb{R})$. Let $V$ be the conformal factor defined by $g_{1}=$ $V g_{1 / 2}$. Near a conical point, $V$ equals

$$
V=e^{-2 v} \frac{4 n_{j}^{2}|z|^{n_{j}-1}}{\left(1+|z|^{2 n_{j}}\right)^{2}} .
$$

By hypothesis, $n_{j} \geq 2$ and $n_{j} \in \mathbb{Z}$. Hence $V$ vanishes precisely at the conical points, and the metric $g:=V^{-1} g_{1 / 2}$ is smooth on $M$, including at the points $P_{j}$ where it reads

$$
g=\frac{1}{4} n_{j}^{-2} e^{4 v}\left(1+|z|^{2 n_{j}}\right)^{-2}|d z|^{2} .
$$

By Lemma 2.3, $g$ satisfies the Ricci condition outside the isolated zeros of $V$ and so it is a Ricci metric on $M$. 
Using this result, we can give more examples of Ricci metrics on compact Riemann surfaces.

Corollary 6.3. Let $M$ be a compact Riemann surface of genus $g$, and $\phi: M \rightarrow \mathbb{S}^{2}$ a branched cover of degree $n=g-1$. Then $M$ admits Ricci metrics.

Proof. Pull back the spherical metric from $\mathbb{S}^{2}$ to $M$ via $\phi$, i.e., $g_{1}:=\phi^{*} g_{\text {sph. }}$ Every branching point of order $n_{j}$ becomes a conical point of $\left(M, g_{1}\right)$ of angle $2 \pi n_{j}$. By the Riemann-Hurwitz formula,

$$
-\sum_{j=1}^{k}\left(n_{j}-1\right)+2 n=2-2 g
$$

hence the conical angles of $g_{1}$ satisfy the constraint (6.3) if and only if $n=g-1$. It follows from Proposition 6.2 that for covers of this degree, the surface $M$ admits Ricci metrics.

By composing $\phi$ with a conformal transformation of $\mathbb{S}^{2}$ which is not an isometry (an element in $\mathrm{PSL}_{2}(\mathbb{C}) \backslash \mathrm{SO}_{3}$ ) we obtain another Ricci metric, hence Ricci metrics arising from branched coverings are not unique in their conformal class.

Generically, a surface $M$ of genus $g$ does not admit branched coverings over $\mathbb{S}^{2}$ of degree $n \leq g-1$ with a branching point of order $n, c f$. [7].

Example 6.4. Let $M$ be a hyperelliptic Riemann surface of odd genus. Then $M$ admits Ricci metrics. Indeed, if $\phi: M \rightarrow \mathbb{S}^{2}$ is a branched double cover, then $\phi^{(g-1) / 2}$ is a branched cover of degree $g-1$ and we can apply Corollary 6.3.

\subsection{An explicit Ricci metric with one zero for the Gauss curvature in every genus $g \geq 2$}

We give below a different way of constructing compact Ricci surfaces of every genus $g \geq 2$, which shows that there are definitely more Ricci surfaces than triply periodic minimal surfaces.

Let $M$ be a closed oriented topological surface of genus $g \geq 1$. Fix a homology basis consisting of $2 g$ simple closed curves $\alpha_{1}, \ldots, \alpha_{2 g}$ such that $\alpha_{j}$ is disjoint from $\alpha_{i}$ unless $\{i, j\}=\{2 k-1,2 k\}$ for some $k \in\{1, \ldots, g\}$, and $\alpha_{2 k-1}$ meets $\alpha_{2 k}$ in precisely one point. Choose a point $p \in M$ and choose simple loops $\gamma_{j}$ freely homotopic to $\alpha_{j}$ such that they meet only in $p$. By cutting along $\gamma_{j}$, we obtain a $4 g$-gon $Q$ with vertices $P_{1}, \ldots, P_{4 g}$. To recover $M$, one must identify in $Q$ the pairs of sides $\gamma_{j}^{\prime}$ and $\gamma_{j}^{\prime \prime}$ corresponding to the cut along $\gamma_{j}$.

By joining $P_{1}$ with $P_{3}, \ldots, P_{4 g-1}$ we obtain a (combinatorial) decomposition of $Q$ into triangles. To define a spherical metric on $M$ it is enough to endow each of these triangles with the structure of a spherical triangle with geodesic sides, and then glue them in the obvious way provided that the lengths of $\gamma_{j}^{\prime}$ and $\gamma_{j}^{\prime \prime}$ coincide. A basic remark is that the result of such a gluing is a smooth spherical metric along the interiors of the edges. In the unique vertex $P$, we get a conical point of total angle 
equal to sum of the angles of the $4 g-2$ triangles. We get moreover a conformal structure on $M$, since the singularity of the conformal structure at $P$ is removable.

Example 6.5. For every $g \geq 2$ and for every $\pi(4 g-2)<\theta<5 \pi(4 g-2)$ there exists at least one spherical metric on a surface of genus $g$ with a unique conical point of angle $\theta$. We construct it by requiring the $4 g-2$ triangles in the $4 g$-gon $Q$ to be equilateral (and congruent) of angle $\alpha=\frac{\theta}{3(4 g-2)}$ (an equilateral spherical triangle of angle $\alpha$ exists for every $\alpha \in(\pi / 3,5 \pi / 3))$. In particular, by choosing $\alpha=\pi \frac{4 g-3}{6 g-3}$, the conical angle becomes $2 \pi(4 g-3)$, and so the hypothesis of Proposition 6.2 holds.

In general, there are $12 g-6$ edges which must be identified in pairs, hence $6 g-3$ parameters giving the lengths of the edges. In each triangle, the edges $e_{1}, e_{2}, e_{3}$ must satisfy a spherical triangle inequality of the form

$$
e_{1}+e_{2}>\min \left\{e_{3}, 2 \pi-e_{3}\right\}
$$

We want to prescribe the conical angle at $P$ to be equal to $2 \pi(4 g-3)$. There seem therefore to be $6 g-4$ degrees of freedom for this construction. This coincides with the dimension of the total space of the tautological fibration over the Teichmüller space of $M$, which is a surface fibration of fiber $(M, c)$ over the conformal structure $c$. Fixing the conical point $P$ amounts to choosing a point in the fiber. So we conjecture that in every conformal class on $M$ and for every point $P \in M$ there exists a spherical metric on $M$ with a conical singularity at $P$ of angle $2 \pi(4 g-3)$.

An existence result for spherical conical metrics was proved by Troyanov [24], but it does not cover the case needed here. Indeed, when there exists a unique conical point, in [24, Theorem C] requires the angle to be comprised strictly between $\pi(4 g-2)$ and $\pi(4 g+2)$. The upper bound is due to the explicit Trudinger constant $4 \pi$ in the Trudinger-Sobolev inequalities. Thus we cannot so far prove that in every conformal class there exist spherical metrics, but we can at least construct one Ricci surface in every genus $g \geq 2$ with curvature vanishing at precisely one point.

Theorem 6.6. For every $g \geq 2$ there exists an oriented closed surface of genus $g$ with a Ricci metric whose curvature vanishes precisely at one point, to order $8 g-1$.

Proof. Apply Proposition 6.2 to the spherical metric on $M$ with one conical point of angle $2 \pi(4 g-3)$ constructed in Example 6.5.

\subsection{Conical Ricci metrics of positive curvature}

A metric on the unit disk $\mathbb{D}$ is called conical at $z_{0} \in \mathbb{D}$ of angle $\alpha \in \mathbb{R}$ if it is of the form $g=\left|z-z_{0}\right|^{\frac{\alpha}{\pi}-2} h$ where $h$ is a smooth conformal metric. This definition extends directly to Riemann surfaces. 
Theorem 6.7. Let $M$ be a Riemann surface of genus $g \geq 2, P_{1}, \ldots, P_{k} \in M$ marked points and $\alpha_{j}=2 \pi n_{j}$ prescribed real angles satisfying

$$
\sum_{j=1}^{k}\left(\alpha_{j}-2 \pi\right)=2 \pi(4 g-4) .
$$

Then there exists a positively curved Ricci metric on $M \backslash\left\{P_{1}, \ldots, P_{k}\right\}$ with conical singularity of angle $\alpha_{j}$ at $P_{j}$ for all $j=1, \ldots, k$.

Proof. Let $g_{-1}$ be the unique smooth hyperbolic metric in the conformal class of $M$ given by the Riemann uniformization theorem. The hypothesis (6.4) on the angles implies

$$
\sum_{j=1}^{k}\left(\pi+\frac{\alpha_{j}}{2}-2 \pi\right)=2 \pi(2 g-2),
$$

so Lemma 6.1 gives us a conical flat metric $g_{0}$ on $M$ with cone angle $\pi+\frac{\alpha_{j}}{2}$ at $P_{j}$. Let $V$ be the conformal factor such that $g_{0}=V g_{-1}$. Then by Lemma 2.5 the metric defined by $g_{R}:=V g_{0}$ is Ricci outside the conical points, with positive Gaussian curvature $K=V^{2}$. Near $P_{j}, V$ is by construction of the form $|z|^{\frac{\alpha_{j}}{2 \pi}-1}$ times a smooth function on $M$, so the metric $g_{R}=V^{2} g_{-1}$ is conical on $M$ in the sense of our definition, of angle $\alpha_{j}$ at $P_{j}$.

More generally, in a given conformal class with marked points, there exist unique hyperbolic metrics of prescribed conical singularities (see [24, Theorem A]). The condition (6.4) in the above theorem can therefore be relaxed. By the same argument, we can construct conical Ricci metrics of non-positive curvature. However we do not have a definitive answer to the uniqueness question, so we leave open the classification of conical Ricci metrics.

\section{Appendix}

\section{A. Link with the Weierstrass-Enneper parametrization}

We adopted in this paper the viewpoint of differential geometry. There exists an alternate local description of minimal surfaces, found by Enneper and Weiertrass, as being governed by 3 holomorphic functions with certain additional properties. In this appendix we show how to translate some of our preliminary results in the language of the Weierstrass-Enneper parametrization.

Let $A: \Omega \rightarrow \mathbb{R}^{3}$ be an isothermal parametrization of a surface $(M, g) \subset \mathbb{R}^{3}$. This means that the vector fields $A_{x}:=\partial_{x}, A_{y}:=\partial_{y}$ are mutually orthogonal and of equal length:

$$
\left|A_{x}\right|=\left|A_{y}\right|=e^{-f}, \quad\left\langle A_{x}, A_{y}\right\rangle=0
$$


and so the (pull-back by $A$ of the) metric on $M$ inherited from $\mathbb{R}^{3}$ is given by $g=e^{-2 f}|d z|^{2}$. The second fundamental form is computed in terms of the unitlength normal field $v=e^{2 f} A_{x} \times A_{y}$ :

$$
\langle W(X), Y\rangle=\langle X(Y), v\rangle
$$

for every vector fields $X, Y$ tangent to $M$. In the basis $\partial_{x}, \partial_{y}$,

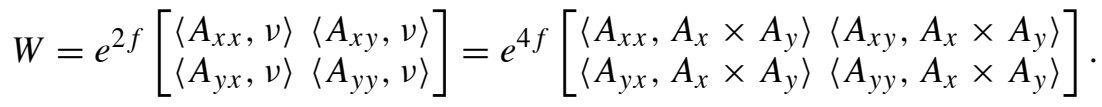

We deduce

$$
\operatorname{tr}(W)=e^{2 f}\left(\left\langle W\left(A_{x}\right), A_{x}\right\rangle+\left\langle W\left(A_{y}\right), A_{y}\right\rangle\right)=e^{2 f}\left\langle A_{x x}+A_{y y}, v\right\rangle .
$$

Notice that the tangential component of $A_{x x}+A_{y y}$ vanishes (we compute $\nabla_{\partial_{x}} \partial_{x}=$ $f_{y} \partial_{y}-f_{x} \partial_{x}=-\nabla_{\partial_{y}} \partial_{y}$ for the Levi-Civita connection on $M$ ). Hence, $M$ is minimal if and only if $A$ is harmonic. From now on we assume this to be the case.

Let $C:=A_{x}-i A_{y}=2 \partial_{z} A$. Since $A$ is harmonic, the $\mathbb{C}^{3}$-valued function $C$ must be holomorphic. Moreover, if $\langle\cdot, \cdot\rangle$ denotes the $\mathbb{C}$-bilinear extension of the standard scalar product on $\mathbb{R}^{3}$, the identities (A.1) encoding the fact that $A$ is an isothermal parametrization mean precisely

$$
\langle C, C\rangle=0, \quad\langle C, \bar{C}\rangle=2 e^{-2 f} \text {. }
$$

Define a complex-valued function from the coefficients of $W$ :

$$
h:=e^{-2 f}\left(W_{11}-i W_{12}\right)=e^{2 f}\left\langle A_{x x}-i A_{x y}, A_{x} \times A_{y}\right\rangle .
$$

Lemma A.1. The function $h$ is holomorphic.

Proof. We can re-write $h$ as

$$
\begin{aligned}
h & =e^{2 f}\left\langle C^{\prime}, A_{x} \times A_{y}\right\rangle=e^{2 f}\left\langle C^{\prime}, C \times A_{y}\right\rangle=e^{2 f}\left\langle C^{\prime}, C \times \frac{1}{i}\left(\partial_{\bar{z}} A-\partial_{z} A\right)\right\rangle \\
& =\frac{1}{2 i} e^{2 f}\left\langle C^{\prime}, C \times \bar{C}\right\rangle=\frac{1}{2 i} e^{2 f}\left\langle C^{\prime} \times C, \bar{C}\right\rangle .
\end{aligned}
$$

Let us show that $\partial_{\bar{z}} h=0$. Since $C$ and $C^{\prime}$ are holomorphic, it is enough to show

$$
\left\langle C^{\prime} \times C, \partial_{\bar{z}}\left(e^{2 f} \bar{C}\right)\right\rangle=0
$$

For this, note the orthogonality relations (always with respect to the complexified inner product)

$$
\langle C, C\rangle=0, \quad\left\langle C, C^{\prime}\right\rangle=0,
$$


the second one being deduced from the first by applying $\partial_{z}$. Also, from

$$
\langle C, \bar{C}\rangle=2 e^{-2 f}
$$

we get, applying $\partial_{\bar{z}}$,

$$
\left\langle C, \overline{C^{\prime}}\right\rangle=-4 f_{z} e^{-2 f}
$$

and so

$$
\left\langle C, \overline{C^{\prime}}+2 f_{z} \bar{C}\right\rangle=0
$$

We have found three vectors $\left(C, C^{\prime}\right.$ and $\left.\overline{C^{\prime}}+2 f_{z} \bar{C}\right)$ orthogonal to $C$, they must therefore be linearly dependent since the complexified inner product is non-degenerate. This implies the vanishing (A.3).

Since $W$ is trace-free and symmetric we $\operatorname{get} \operatorname{det}(W)=-W_{11}^{2}-W_{12}^{2}=$ $-e^{4 f}|h|^{2}$. By the Gauss equation, the curvature of $g$ equals $K=\operatorname{det}(W)$.

On the other hand, since $g=e^{-2 f}|d z|^{2}$, we get $K=-e^{2 f} \Delta f$. Therefore $e^{-2 f} \Delta f$ equals the norm squared of the holomorphic function $h$.

We are now in position to compute $h$ in terms of the Weierstrass-Enneper representation of $A$, exploiting the fact that $C=2 \partial_{z} A$ is holomorphic and isotropic for the complexified inner product. We assume that $A_{x}, A_{y}$ are linearly independent (since they are the tangent vector fields to $M$ in a chart). Write $C=(a, b, c)$ with holomorphic components $a, b, c$. We claim that since $a^{2}+b^{2}+c^{2}=\langle C, C\rangle=0$, there exist holomorphic functions $\alpha, \beta$ such that

$$
a=\alpha\left(1+\beta^{2}\right), \quad b=i \alpha\left(1-\beta^{2}\right), \quad c=2 i \alpha \beta .
$$

To this end, set $\alpha:=\frac{a-i b}{2}$ and $\beta=\frac{c}{i a+b}$. It is immediate (using $c^{2}=-(a+$ $i b)(a-i b))$ that (A.7) holds. Furthermore,

Lemma A.2. The holomorphic function $h$ is given by

$$
h=-2 i \alpha \beta^{\prime} .
$$

Proof. We use the expression (A.2) for $h$. From (A.5),

$$
\begin{aligned}
2 e^{-2 f} & =|a|^{2}+|b|^{2}+|c|^{2} \\
& =|\alpha|^{2}\left(\left|1+\beta^{2}\right|^{2}+\left|1-\beta^{2}\right|^{2}+4|\beta|^{2}\right) \\
& =2|\alpha|^{2}\left(1+|\beta|^{2}\right)^{2} .
\end{aligned}
$$


Next, we write using determinants

$$
\left\langle C^{\prime}, C \times \bar{C}\right\rangle=\left|\begin{array}{ccc}
\alpha\left(1+\beta^{2}\right) & \bar{\alpha}\left(1+\bar{\beta}^{2}\right) & \alpha^{\prime}\left(1+\beta^{2}\right)+2 \alpha \beta^{\prime} \beta \\
i \alpha\left(1-\beta^{2}\right) & -i \bar{\alpha}\left(1-\bar{\beta}^{2}\right) & i \alpha^{\prime}\left(1-\beta^{2}\right)-2 i \alpha \beta^{\prime} \beta \\
2 i \alpha \beta & -2 i \bar{\alpha} \bar{\beta} & 2 i \alpha^{\prime} \beta+2 i \alpha \beta^{\prime}
\end{array}\right|
$$

In the third column, the first terms form a multiple (namely, $\alpha^{\prime} / \alpha$ times) the first column, hence they do not contribute to the determinant. We extract $\alpha, \bar{\alpha}$, respectively $\alpha \beta^{\prime}$ which are common factors in the first, second, respectively third column. We also extract $i$, respectively $2 i$ as common factors in the second, respectively third line. We are left with

$$
\left\langle C^{\prime}, C \times \bar{C}\right\rangle=-2|\alpha|^{2} \alpha \beta^{\prime}\left|\begin{array}{ccc}
1+\beta^{2} & 1+\bar{\beta}^{2} & 2 \beta \\
1-\beta^{2} & -\left(1-\bar{\beta}^{2}\right) & -2 \beta \\
\beta & -\bar{\beta} & 1
\end{array}\right|
$$

The above determinant yields (after adding the second line to the first for simplicity)

$$
2\left(-|\beta|^{2}-1-\bar{\beta}^{2}\left(1+\beta^{2}\right)\right)=-2\left(1+|\beta|^{2}\right)^{2}
$$

Gathering the above formulas we get the lemma.

\section{References}

[1] L. AlíAS and B. PALMER, A duality result between the minimal surface equation and the maximal surface equation, An. Acad. Brasil. Ciênc. 73 (2001), 161-164.

[2] C. BÄR, P. GAUdUCHON and A. MoroiAnU, Generalized cylinders in semi-Riemannian and spin geometry, Math. Z. 249 (2005), 545-580.

[3] A. BESSE, "Einstein Manifolds", Ergebnisse der Mathematik und ihrer Grenzgebiete (3), Vol. 10, Springer-Verlag, Berlin, 1987.

[4] W. BlaschKe and H. REICHARDT, "Einführung in die Differentialgeometrie", Grundlehren math. Wissenschaften, Vol. 58, Springer-Verlag, Berlin, 1960.

[5] T. Colding and W. Minicozzi II, "A Course in Minimal Surfaces", Graduate Studies in Mathematics, Vol. 121, AMS, Providence, RI, 2011.

[6] A. Edmonds, R. KUlKARNi and R. STONG, Realizability of branched coverings of surfaces, Trans. Amer. Math. Soc. 282 (1984), 773-790.

[7] H. FARKAS, Special divisors on compact Riemann surfaces, Proc. Amer. Math. Soc. 19 (1968), 315-318.

[8] TH. FRIEDRICH, On the spinor representation of surfaces in Euclidean 3-space, J. Geom. Phys. 28 (1998), 143-157.

[9] H. FURUHATA, An intrinsic characterization of isometric pluriharmonic immersions with codimension one, J. Geom. 65 (1999), 111-116.

[10] Q. HAN, J.-X. Hong and C.-S. LIN, Local isometric embedding of surfaces with nonpositive Gaussian curvature, J. Differential Geom. 63 (2003), 475-520. 
[11] H. KARCHER, The triply periodic minimal surfaces of Alan Schoen and their constant mean curvature companions, Manuscripta Math. 64 (1989), 291-357.

[12] J. KAZDAN and F. WARNER, Existence and conformal deformation of metrics with prescribed Gaussian and scalar curvatures, Ann. of Math. (2) 101 (1975), 317-331.

[13] H. B. LAWSON JR., Some intrinsic characterizations of minimal surfaces, J. Anal. Math. 24 (1971), 151-161.

[14] B . MALGRANGE, Existence et approximation des solutions des équations aux dérivées partielles et des équations de convolution, Ann. Inst. Fourier (Grenoble) 6 (1955-1956), 271355.

[15] W. MEEKS III, The theory of triply periodic minimal surfaces, Indiana Univ. Math. J. 39 (1990), 877-936.

[16] S. MOROIANU, Uniformization of $S^{2}$ and flat singular surfaces, arXiv:1101.2355.

[17] M. PINL, Über einen Satz von G. Ricci-Curbastro und die Gausssche Krümmung der Minimalflächen, Arch. Math. 4 (1953), 369-373.

[18] G. RicCi-Curbastro, Sulla teoria intrinseca delle superficie ed in ispecie di quelle di secondo grado, Atti R. Ist. Ven. di Lett. ed Arti 6 (1895), 445-488.

[19] M. SAKAKI, On minimal surfaces with the Ricci condition in space forms, Kodai Math. J. 17 (1994), 452-455.

[20] A. SCHOEN, Infinite periodic minimal surfaces without selfintersections, NASA Technical Note No. TN D-5541 (1970).

[21] C. H. Taubes, Minimal Surfaces in Germs of Hyperbolic 3-Manifolds, In: "Proceedings of the Casson Fest", Geom. Topol. Monogr., Vol. 7, Geom. Topol. Publ., Coventry, 69-100.

[22] M. Traizet, On the genus of triply periodic minimal surfaces, J. Differential Geom. 79 (2008), 243-275.

[23] M. Troyanov, Les surfaces euclidiennes à singularités coniques, Enseign. Math. (2) 32 (1986), 79-94.

[24] M. TRoyanov, Prescribing curvature on compact surfaces with conical singularities, Trans. Amer. Math. Soc. 324 (1991), 793-821.

[25] T. Vlachos, Minimal surfaces in a sphere and the Ricci condition, Ann. Global Anal. Geom. 17 (1999), 129-150.

[26] T. VLACHOS, Intrinsic obstructions to the existence of isometric minimal immersions, $\mathrm{Pa}$ cific J. Math. 205 (2002), 491-510.

[27] M. WEBER and M. WOLF, Teichmüller theory and handle addition for minimal surfaces, Ann. of Math. (2) 156 (2002), 713-795.

[28] S.-T. YAU, Problem section, In: "Seminar on Differential Geometry", Ann. Math. Stud., Vol. 102, Princeton Univ. Press, Princeton, 1982, 669-706.

[29] S.-T. YAU, Open problems in geometry, In: "Chern - a Great Geometer of the Twentieth Century”, Internat. Press, Hong Kong, 1992, 275-319.

Université de Versailles-St Quentin

Laboratoire de Mathématiques

UMR 8100 du CNRS

45 avenue des États-Unis

78035 Versailles, France

andrei.moroianu@math.cnrs.fr

Institutul de Matematică al Academiei Române

P.O. Box 1-764

RO-014700 Bucharest, Romania

sergiu.moroianu@imar.ro 